\title{
Algorithm for the numerical calculation of the serial components of the normal form of depolarizing Mueller matrices
}

\author{
IGNACIO SAN JOSÉ, ${ }^{1}$ JOSÉ J. GIL, ${ }^{2, *}$ R. OSSIKOVSKI, ${ }^{3}$ \\ ${ }^{1}$ Instituto Aragonés de Estadística, Gobierno de Aragón, Bernardino Ramazzini 5, 50015 Zaragoza, Spain \\ ${ }^{2}$ Department of Applied Physics, University of Zaragoza, Pedro Cerbuna 12, 50009 Zaragoza, Spain \\ ${ }^{3}$ LPICM, CNRS, Ecole Polytechnique, Institut Polytechnique de Paris, 91128 Palaiseau, France
}

*Corresponding author: ppgil@unizar.es

The normal form of a depolarizing Mueller matrix constitutes an important tool for the phenomenological interpretation of experimental polarimetric data. Due to its structure as a serial combination of three Mueller matrices, namely a canonical depolarizing Mueller matrix sandwiched between two pure (nondepolarizing) Mueller matrices, it overcomes the necessity of making a priori choices on the order of the polarimetric components, as this occurs in other serial decompositions. Because Mueller polarimetry addresses more and more applications in a wide range of areas in science, engineering, medicine, etc., the normal form decomposition has an enormous potential for the analysis of experimentally determined Mueller matrices. However, its systematic use has been limited to some extent because of the lack of numerical procedure for the calculation of each polarimetric component, in particular in the case of type-II Mueller matrices. In this work, an efficient algorithm applicable to the decomposition of both type-II and type-I Mueller matrices is presented.

\section{INTRODUCTION}

Nowadays Mueller polarimetry is a well-known technique involving both experimental and theoretical tools that allow for the determination and the analysis of a number of physical and optical properties of a great variety of material samples. The intricate mathematical structure of Mueller matrices makes it necessary for appropriate theoretical approaches (essentially serial and parallel decomposition theorems [1]) to be implemented in the form of numerical algorithms in order to process efficiently the experimental data.

A particularly interesting theoretical approach for the analysis of Mueller matrices is the so-called normal form [2-4], according to which any depolarizing Mueller matrix can be expressed as a product of three Muller matrices, namely a canonical depolarizing Mueller matrix [5] sandwiched between two nondepolarizing
Mueller matrices. The normal form is intimately related to the symmetric decomposition of depolarizing Mueller matrices [4], as discussed in the end of section 3.

The main purpose of the present paper is to present an efficient algorithm for the numerical calculation of the Mueller matrix components of the normal form for both type-I and type-II experimental Mueller matrices. Before proceeding, we introduce a brief summary of the terminology, notations and concepts that will be used along the paper.

Following the common practice, we use the term nondepolarizing (also pure or Mueller-Jones) for Mueller matrices associated with media that preserve the degree of polarization for any incoming fully polarized light, while the term depolarizing (or nonpure) is used to refer to Mueller matrices that do not. To specify that a given Mueller matrix is pure, we denote it as $\mathbf{M}_{J}$ (with the subscript J).

Any Mueller matrix can be conveniently cast into the partitioned form [6]

$$
\begin{gathered}
\mathbf{M}=m_{00} \hat{\mathbf{M}}, \quad \hat{\mathbf{M}} \equiv\left(\begin{array}{cc}
1 & \mathbf{D}^{T} \\
\mathbf{P} & \mathbf{m}
\end{array}\right), \\
\mathbf{m} \equiv \frac{1}{m_{00}}\left(\begin{array}{lll}
m_{11} & m_{12} & m_{13} \\
m_{21} & m_{22} & m_{23} \\
m_{31} & m_{32} & m_{33}
\end{array}\right), \\
\mathbf{D} \equiv \frac{\left(m_{01}, m_{02}, m_{03}\right)^{T}}{m_{00}}, \mathbf{P} \equiv \frac{\left(m_{10}, m_{20}, m_{30}\right)^{T}}{m_{00}},
\end{gathered}
$$

where the superscript $T$ indicates transposition, $m_{00}$ is the mean intensity coefficient (MIC) and $\mathbf{D}$ and $\mathbf{P}$ are the respective diattenuation and polarizance vectors of $\mathbf{M}$. The absolute values of these vectors are the diattenuation $D \equiv|\mathbf{D}|$ and the polarizance $P \equiv|\mathbf{P}|$ [7].

Any Mueller matrix $\mathbf{M}$ can be put in a one-to-one correspondence with its associated covariance matrix $\mathbf{H}$ defined as $[8]$

$$
\mathbf{H}(\mathbf{M})=\frac{1}{4} \sum_{i, j=0}^{3} m_{i j}\left(\boldsymbol{\sigma}_{i} \otimes \boldsymbol{\sigma}_{j}\right)
$$


where $\boldsymbol{\sigma}_{i}$ are the Pauli spin matrices (taken in the order commonly used in polarization optics),

$$
\boldsymbol{\sigma}_{0}=\left(\begin{array}{ll}
1 & 0 \\
0 & 1
\end{array}\right), \boldsymbol{\sigma}_{1}=\left(\begin{array}{cc}
1 & 0 \\
0 & -1
\end{array}\right), \boldsymbol{\sigma}_{2}=\left(\begin{array}{ll}
0 & 1 \\
1 & 0
\end{array}\right), \boldsymbol{\sigma}_{3}=\left(\begin{array}{cc}
0 & -i \\
i & 0
\end{array}\right) .
$$

Leaving aside passivity constraints [9], a real $4 \times 4$ matrix is a Mueller matrix if and only if the Hermitian matrix $\mathbf{H}$ is positivesemidefinite (i.e., the four eigenvalues of $\mathbf{H}$ are nonnegative). If the Mueller matrix $\mathbf{M}$ is pure, then all but one eigenvalues of $\mathbf{H}$ vanish The elements of $\mathbf{M}$ can be expressed through those of $\mathbf{H}$ as follows

$$
m_{i j}=\operatorname{tr}\left[\left(\boldsymbol{\sigma}_{i} \otimes \boldsymbol{\sigma}_{j}\right) \mathbf{H}\right] .
$$

Let us also recall that any unitary similarity transformation of $\mathbf{H}$ $\mathbf{V H} \mathbf{V}^{\dagger}$ with $\mathbf{V}^{\dagger}=\mathbf{V}^{-1}$ (V is an arbitrary unitary matrix), constitutes an alternative positive semidefinite Hermitian matrix that also contains all the polarimetric information of the medium, and therefore, can be likewise used to describe the latter. Among these possible covariance matrices, it is sometimes useful to consider the so-called coherency matrix $\mathbf{C}$ [8], linked to $\mathbf{H}$ through the similarity transformation

$$
\begin{aligned}
& \mathbf{C}(\mathbf{M})=\mathcal{L}[\mathbf{H}(\mathbf{M})] \mathcal{L}^{-1}, \\
& \mathcal{L} \equiv \frac{1}{\sqrt{2}}\left(\begin{array}{cccc}
1 & 0 & 0 & 1 \\
1 & 0 & 0 & -1 \\
0 & 1 & 1 & 0 \\
0 & i & -i & 0
\end{array}\right) .
\end{aligned}
$$

A physically significant parameter is the rank $r$ of the covariance (or coherency) matrix, $\operatorname{rank}(\mathbf{C})=\operatorname{rank}(\mathbf{H}) \equiv r$, which determines the minimum number of pure parallel components of $\mathbf{M}[10,11]$. The explicit expressions for $\mathbf{H}(\mathbf{M}), \mathbf{M}(\mathbf{H}), \mathbf{C}(\mathbf{M})$ and $\mathbf{M}(\mathbf{C})$ can be found in Refs. [1,12].

Given a Mueller matrix $\mathbf{M}$, its normal form decomposition follows from the algebraic properties of its associated N-matrix , defined as $\mathbf{N} \equiv \mathbf{G} \mathbf{M}^{T} \mathbf{G} \mathbf{M}$, where the matrix $\mathbf{G}$ is the Minkowski metrics, $\mathbf{G} \equiv \operatorname{diag}(1,-1,-1,-1)[2-4,13-15]$. The matrix $\mathbf{N}$ is said to be diagonalizable if there exists an invertible matrix $\mathbf{A}$ such that $\mathbf{A}^{-1} \mathbf{N A}$ is diagonal. Type-I Mueller matrices are those whose associated N-matrix is diagonalizable, while a Mueller matrix is of type-II if and only if its associated N-matrix is not diagonalizable $[4,5]$.

Leaving aside the fact that the diagonalizability test is dealt with in a large number of linear algebra treatises, it is worth recalling that Gopala Rao et al. [15], introduced a simple criterion to distinguish between type-I and type-II Mueller matrices, based on the inspection of the properties of the N-matrices $\mathbf{N} \equiv \mathbf{G} \mathbf{M}^{T} \mathbf{G} \mathbf{M}$ and $\mathbf{N}^{\prime} \equiv \mathbf{G} \mathbf{M ~ G} \mathbf{M}^{T}$. In particular, if $\mathbf{N} \neq \mathbf{0}, \mathbf{N}^{\prime} \neq \mathbf{0}$, and both $\mathbf{N}$ and $\mathbf{N}^{\prime}$ have respective totally polarized eigenstates, then $\mathbf{M}$ is of type II, while $\mathbf{M}$ is of type I in all remaining cases.

Given a Mueller matrix $\mathbf{M}$, its normal form decomposition is formulated as

$$
\mathbf{M}=\mathbf{M}_{J 2} \mathbf{M}_{\Delta} \mathbf{M}_{J 1}
$$

where $\mathbf{M}_{J 1}$ and $\mathbf{M}_{J 2}$ are pure Mueller matrices while the depolarizer matrix $\mathbf{M}_{\Delta}$ takes one of the two following canonical forms $\mathbf{M}_{\Delta d}$ and $\mathbf{M}_{\Delta n d}$ depending on whether $\mathbf{M}$ is of type I or type II [5],

$$
\begin{gathered}
\mathbf{M}_{\Delta d} \equiv \operatorname{diag}\left(d_{0}, d_{1}, d_{2}, \varepsilon d_{3}\right), \\
0 \leq d_{3} \leq d_{2} \leq d_{1} \leq d_{0}, \varepsilon \equiv(\operatorname{det} \mathbf{M}) / \operatorname{det} \mathbf{M} \mid
\end{gathered}
$$

$$
\mathbf{M}_{\Delta n d} \equiv\left(\begin{array}{cccc}
2 a_{0} & -a_{0} & 0 & 0 \\
a_{0} & 0 & 0 & 0 \\
0 & 0 & a_{2} & 0 \\
0 & 0 & 0 & a_{2}
\end{array}\right), 0 \leq a_{2} \leq a_{0}
$$

where $\left(d_{0}, d_{1}, d_{2}, d_{3}\right)$ and $\left(a_{0}, a_{0}, a_{2}, a_{2}\right)$ are the square roots of the (nonnegative) eigenvalues $\left(\rho_{0}, \rho_{0}, \rho_{2}, \rho_{2}\right)$ of the $\mathrm{N}$-matrix $\mathbf{N}$ associated with $\mathbf{M}$ for type-I and type-II Mueller matrices respectively.

The normal form decomposition of a type-II Mueller matrix can be transformed to the following form $[1,5,13]$, which will be useful un further sections

$$
\begin{gathered}
\mathbf{M}=\mathbf{M}_{J B} \mathbf{M}_{\Delta I I} \mathbf{M}_{J A} \\
\mathbf{M}_{\Delta I I} \equiv\left(\begin{array}{cccc}
a & b-a & 0 & 0 \\
0 & b & 0 & 0 \\
0 & 0 & a_{2} & 0 \\
0 & 0 & 0 & a_{2}
\end{array}\right),(a>b)
\end{gathered}
$$

where the new components can be calculated from the former ones by means of

$$
\mathbf{M}=\left(\mathbf{M}_{J 2} \mathbf{M}_{D a} \mathbf{M}_{D b}\right)\left(\mathbf{M}_{D b}^{-1} \mathbf{M}_{D a}^{-1} \mathbf{M}_{\Delta n d} \mathbf{M}_{D a}\right)\left(\mathbf{M}_{D a}^{-1} \mathbf{M}_{J 1}\right),
$$

where

$$
\begin{aligned}
& \mathbf{M}_{\Delta I I}=\mathbf{M}_{D b}^{-1} \mathbf{M}_{D a}^{-1} \mathbf{M}_{\triangle n d} \mathbf{M}_{D a}, \\
& \mathbf{M}_{J B}=\mathbf{M}_{J 2} \mathbf{M}_{D a} \mathbf{M}_{D b}, \\
& \mathbf{M}_{J A}=\mathbf{M}_{D a}^{-1} \mathbf{M}_{J 1},
\end{aligned}
$$

with

$$
\begin{gathered}
\mathbf{M}_{D a} \equiv \frac{1}{1+c_{\kappa a}} \hat{\mathbf{M}}_{D a+}, \quad \mathbf{M}_{D a}^{-1} \equiv \frac{1}{1-c_{\kappa a}} \hat{\mathbf{M}}_{D a-} \\
\mathbf{M}_{D b} \equiv \frac{1}{S_{\kappa b}} \hat{\mathbf{M}}_{D b+}, \quad \mathbf{M}_{D b}^{-1} \equiv \frac{1}{S_{\kappa b}} \hat{\mathbf{M}}_{D b-} \\
\hat{\mathbf{M}}_{D i+} \equiv\left(\begin{array}{cccc}
1 & \mathrm{c}_{\kappa i} & 0 & 0 \\
\mathrm{c}_{\kappa i} & 1 & 0 & 0 \\
0 & 0 & S_{\kappa i} & 0 \\
0 & 0 & 0 & S_{\kappa i}
\end{array}\right), \hat{\mathbf{M}}_{D i-} \equiv\left(\begin{array}{cccc}
1 & -\mathrm{c}_{\kappa i} & 0 & 0 \\
-\mathrm{c}_{\kappa i} & 1 & 0 & 0 \\
0 & 0 & S_{\kappa i} & 0 \\
0 & 0 & 0 & S_{\kappa i}
\end{array}\right), \\
S_{\kappa i} \equiv \sin \kappa_{i}, c_{\kappa i} \equiv \cos \kappa_{i}, \quad(i=a, b) \\
\cos \kappa_{a} \equiv \frac{3 a-b}{a+b}, \quad \cos \kappa_{b} \equiv \frac{b-a}{a+b} .
\end{gathered}
$$

Note that, since $a>b>0$ the above matrices are always nonsingular.

Once the required theoretical background has been presented, the next sections are devoted to the development of the algorithm for the numerical calculation of the Mueller matrices of the components of the normal form. In the case of type-II Mueller matrices, the algorithm provides the serial matrix components in the form (8) ( $\left.\mathbf{M}_{\triangle I I}, \mathbf{M}_{J A}, \mathbf{M}_{J B}\right)$ which can be easily transformed into $\mathbf{M}_{\Delta n d}, \mathbf{M}_{J 1}$ and $\mathbf{M}_{J 2}$ by means of the expressions 


$$
\begin{gathered}
\mathbf{M}_{\triangle n d}=\mathbf{M}_{D a} \mathbf{M}_{D b} \mathbf{M}_{\triangle I I} \mathbf{M}_{D a}^{-1} \\
\mathbf{M}_{J 2}=\mathbf{M}_{J B} \mathbf{M}_{D b}^{-1} \mathbf{M}_{D a}^{-1} \\
\mathbf{M}_{J 1}=\mathbf{M}_{D a} \mathbf{M}_{J A}
\end{gathered}
$$

\section{BI-DIAGONALIZATION OF THE MUELLER MATRIX}

The procedure starts with the transformation of the Mueller matrix $\mathbf{M}$ into a bi-diagonal Mueller matrix $\mathbf{M}_{B}$ of the form

$$
\mathbf{M}_{B}=\left(\begin{array}{cccc}
b_{00} & b_{01} & 0 & 0 \\
0 & b_{11} & b_{12} & 0 \\
0 & 0 & b_{22} & b_{23} \\
0 & 0 & 0 & b_{33}
\end{array}\right),
$$

which is obtained from $\mathbf{M}$ by its pre- and post-multiplication by two kinds of orthochronous proper Lorentz matrices or OPLMs (i.e., nonsingular pure Mueller matrices [1,15]), denoted as $\mathbf{U}_{i}$ and $\mathbf{V}_{i}$ respectively, that is $\mathbf{M}_{B}=\mathbf{U}_{3} \mathbf{U}_{2} \mathbf{U}_{1} \mathbf{M}_{B} \mathbf{V}_{1} \mathbf{V}_{2}$. Schematically,

$$
\begin{aligned}
& \left(\begin{array}{llll}
\mathrm{x} & \mathrm{x} & \mathrm{x} & \mathrm{x} \\
\mathrm{x} & \mathrm{x} & \mathrm{x} & \mathrm{x} \\
\mathrm{x} & \mathrm{x} & \mathrm{x} & \mathrm{x} \\
\mathrm{x} & \mathrm{x} & \mathrm{x} & \mathrm{x}
\end{array}\right) \rightarrow\left(\begin{array}{llll}
\mathrm{x} & \mathrm{x} & \mathrm{x} & \mathrm{x} \\
0 & \mathrm{x} & \mathrm{x} & \mathrm{x} \\
0 & \mathrm{x} & \mathrm{x} & \mathrm{x} \\
0 & \mathrm{x} & \mathrm{x} & \mathrm{x}
\end{array}\right) \mathbf{V}_{1}\left(\begin{array}{llll}
\mathrm{x} & \mathrm{x} & 0 & 0 \\
0 & \mathrm{x} & \mathrm{x} & \mathrm{x} \\
0 & \mathrm{x} & \mathrm{x} & \mathrm{x} \\
0 & \mathrm{x} & \mathrm{x} & \mathrm{x}
\end{array}\right) \\
& \mathbf{U}_{2}\left(\begin{array}{lllll}
\mathrm{x} & \mathrm{x} & 0 & 0 \\
0 & \mathrm{x} & \mathrm{x} & \mathrm{x} \\
0 & 0 & \mathrm{x} & \mathrm{x} \\
0 & 0 & \mathrm{x} & \mathrm{x}
\end{array}\right) \rightarrow\left(\begin{array}{llll}
\mathrm{x} & \mathrm{x} & 0 & 0 \\
0 & \mathrm{x} & \mathrm{x} & 0 \\
0 & 0 & \mathrm{x} & \mathrm{x} \\
0 & 0 & \mathrm{x} & \mathrm{x}
\end{array}\right) \mathbf{U}_{3}\left(\begin{array}{llll}
\mathrm{x} & \mathrm{x} & 0 & 0 \\
0 & \mathrm{x} & \mathrm{x} & 0 \\
0 & 0 & \mathrm{x} & \mathrm{x} \\
0 & 0 & 0 & \mathrm{x}
\end{array}\right)
\end{aligned}
$$

where $\mathrm{x}$ denotes the nonzero elements (different, in general).

\section{A. Calculation of the matrix $U_{1}$}

Define the vector

$$
\mathbf{v}_{1}=\mathbf{m}_{1}-\sqrt{\mathbf{m}_{1}^{T} \mathbf{G} \mathbf{m}_{1}} \mathbf{e}_{1}
$$

where $\mathbf{m}_{1}$ is the first column of $\mathbf{M}$ and $\mathbf{e}_{1}=(1,0,0,0)^{T}$. Next, we define the hyperbolic Householder matrix

$$
\mathbf{H}_{1}=\mathbf{G}-2 \frac{\mathbf{v}_{1} \mathbf{v}_{1}^{T}}{\mathbf{v}_{1}^{T} \mathbf{G} \mathbf{v}_{1}}
$$

Note that, provided $\mathbf{m}_{1}^{T} \mathbf{G} \mathbf{m}_{1}>0$ (i.e., the medium is not a depolarizing polarizer, that is $P<1[7,17])$, then

$$
\mathbf{v}_{1}^{T} \mathbf{G} \mathbf{v}_{1}=2\left(m_{00} \sqrt{\mathbf{m}_{1}^{T} \mathbf{G} \mathbf{m}_{1}}-\mathbf{m}_{1}^{T} \mathbf{G} \mathbf{m}_{1}\right) \neq 0,
$$

so that the denominator in Eq. (14) is nonzero.

In the case where $\mathbf{m}_{1}^{T} \mathbf{G m}_{1}=0$ (i.e., $P=1$ ) we take $\mathbf{H}_{1}=\mathbf{I}_{4}$ ( $\mathbf{I}_{4}$ being the $4 \times 4$ identity matrix). The four eigenvalues of the coherency matrix associated with the matrix $\mathbf{G H}_{1} \mathbf{G}$ are

$$
\left\{0,0,0, \frac{m_{00}}{\sqrt{\mathbf{m}_{1}^{T} \mathbf{G m}}}\right\},
$$

with $m_{00} / \sqrt{\mathbf{m}_{1}^{T} \mathbf{G m}_{1}}>0$ and therefore, the matrix $\mathbf{G H}_{1} \mathbf{G}$ is a pure Mueller matrix. Let us now take $\mathbf{U}_{1} \equiv \mathbf{G H}_{1} \mathbf{G}$, which has the property that matrix $\mathbf{U}_{1} \mathbf{M}$ has zero elements in its first column, except for the "00" element.

\section{B. Structure of the matrices $V_{1}, V_{2}, U_{2}, U_{3}$}

The matrices $\mathbf{V}_{1}, \mathbf{V}_{2}, \mathbf{U}_{2}, \mathbf{U}_{3}$ are built from certain Householder matrices that are defined below. Consider the following pair of vectors

$$
\mathbf{x}_{2}=\left(x_{0}, x_{1}, x_{2}\right)^{T}, \mathbf{e}_{2}=(1,0,0)^{T},
$$

and define the vector

$$
\mathbf{u}_{2}=\mathbf{x}_{2}+\sqrt{\mathbf{x}_{2}^{T} \mathbf{x}_{2}} \mathbf{e}_{2},
$$

which generates the $3 \times 3$ Householder matrix

$$
\mathbf{Q}_{2}=\mathbf{I}_{3}-2 \frac{\mathbf{u}_{2} \mathbf{u}_{2}^{T}}{\mathbf{u}_{2}^{T} \mathbf{u}_{2}},
$$

that satisfies the following property

$$
\mathbf{Q}_{2} \mathbf{x}_{2}=-\sqrt{\mathbf{x}_{2}^{T} \mathbf{x}_{2}} \mathbf{e}_{2} .
$$

If $\mathbf{u}_{2}=\mathbf{0}$, then we take $\mathbf{Q}_{2}=\mathbf{I}_{3} \quad\left(\mathbf{I}_{3}\right.$ is the $3 \times 3$ identity matrix). Next, define the matrix

$$
\mathbf{H}_{2}=\left(\begin{array}{cc}
1 & 0 \\
0 & \mathbf{Q}_{2}
\end{array}\right)
$$

so that $\mathbf{G H}_{2}$ is a pure Mueller matrix with $\operatorname{det} \mathbf{G H}_{2}=1$.

Analogously, consider the vectors

$$
\mathbf{x}_{3}=\left(x_{0}, x_{1}\right)^{T}, \mathbf{e}_{3}=(1,0)^{T},
$$

and define the vector

$$
\mathbf{u}_{3}=\mathbf{x}_{3}+\sqrt{\mathbf{x}_{3}^{T} \mathbf{x}_{3}} \mathbf{e}_{3},
$$

which generates the $2 \times 2$ Householder matrix

$$
\mathbf{Q}_{3}=\mathbf{I}_{2}-2 \frac{\mathbf{u}_{3} \mathbf{u}_{3}^{T}}{\mathbf{u}_{3}^{T} \mathbf{u}_{3}},
$$

that satisfies the following property

$$
\mathbf{Q}_{3} \mathbf{x}_{3}=-\sqrt{\mathbf{x}_{3}^{T} \mathbf{x}_{3}} \mathbf{e}_{3} .
$$

If $\mathbf{u}_{3}=\mathbf{0}$, then we take $\mathbf{Q}_{3}=\mathbf{I}_{2} \quad\left(\mathbf{I}_{2}\right.$ is the $2 \times 2$ identity matrix). Next, define the matrix

$$
\mathbf{H}_{3}=\left(\begin{array}{cc}
\mathbf{I}_{2} & \mathbf{0}^{T} \\
\mathbf{0} & \mathbf{Q}_{3}
\end{array}\right)
$$

so that $\mathbf{G H}_{3}$ is a pure Mueller matrix with $\operatorname{det} \mathbf{G H} \mathbf{H}_{3}=1$.

\section{Bi-diagonalization procedure}

Once the matrices $\mathbf{U}_{1}, \mathbf{U}_{2}, \mathbf{U}_{3}, \mathbf{V}_{1}, \mathbf{V}_{2}$ have been defined and their properties have been analyzed, we are in a position to formulate the bi-diagonalization procedure:

1. Take the first column $\mathbf{m}_{1}$ of $\mathbf{M}$ and build $\mathbf{U}_{1}=\mathbf{G} \mathbf{H}_{1} \mathbf{G}$;

2. Calculate $\mathbf{M}^{\prime}=\mathbf{U}_{1} \mathbf{M}$;

3. Take the vector $\mathbf{x}_{2}=\left(m_{01}^{\prime}, m_{02}^{\prime}, m_{03}^{\prime}\right)^{T}$ and build $\mathbf{V}_{1}=\mathbf{G} \mathbf{H}_{2}$;

4. Calculate $\mathbf{M}^{\prime \prime}=\mathbf{M}^{\prime} \mathbf{V}_{1}$;

5. Take the vector $\mathbf{x}_{2}=\left(m_{11}^{\prime \prime}, m_{21}^{\prime \prime}, m_{31}^{\prime \prime}\right)^{T}$ and build $\mathbf{U}_{2}=\mathbf{G H}_{2}$;

6. Calculate $\mathbf{M}^{\prime \prime \prime}=\mathbf{U}_{2} \mathbf{M}^{\prime \prime}$; 
7. Take the vector $\mathbf{x}_{3}=\left(m_{12}^{\prime \prime \prime}, m_{13}^{\prime \prime \prime}\right)^{T}$ and build $\mathbf{V}_{2}=\mathbf{G} \mathbf{H}_{3}$;

8. Calculate $\mathbf{M}^{I V}=\mathbf{M}^{\prime \prime \prime} \mathbf{V}_{2}$;

9. Take the vector $\mathbf{x}_{3}=\left(m_{22}^{I V}, m_{23}^{I V}\right)^{T}$ and build $\mathbf{U}_{3}=\mathbf{G H}_{3}$;

10. Calculate the bi-diagonal Mueller matrix $\mathbf{M}_{B}=\mathbf{U}_{3} \mathbf{M}^{I V}$ $=\mathbf{U}_{3} \mathbf{U}_{2} \mathbf{U}_{1} \mathbf{M} \mathbf{V}_{1} \mathbf{V}_{2}$

\section{ITERATIVE PROCEDURE FOR OBTAINING THE PURE COMPONENTS AND THE CANONICAL DEPOLARIZERS OF THE NORMAL FORM}

The next stage of the algorithm consists in applying consecutive OPLM transformations to the bi-diagonal matrix obtained in the previous section. The process is iterated either until the offdiagonal elements of $\mathbf{M}$ are zero (within the numerical tolerance specified), or until the conditions for $\mathbf{M}$ to be transformed into $\mathbf{M}_{\Delta I I}$ are satisfied. In other words, if the process reaches a matrix of the form of $\mathbf{M}_{\Delta I I}$, then $\mathbf{M}$ is of type-II; otherwise, if the process continues to a diagonal matrix, then $\mathbf{M}$ is a type-I Mueller matrix.

The OPLM matrices used at this stage are the Givens matrices $\mathbf{G}_{i}$ described in the Appendix. Givens matrices with odd subscripts act as left-factors, while those with even subscripts act as rightfactors. In this way, we get the transformed matrix $\mathbf{M}_{B}^{\prime}=\mathbf{G}_{6} \mathbf{G}_{4} \mathbf{G}_{2} \mathbf{M}_{B} \mathbf{G}_{1} \mathbf{G}_{3} \mathbf{G}_{5}$.

Schematically,

$$
\begin{aligned}
&\left(\begin{array}{cccc}
\mathrm{x} & \mathrm{x} & 0 & 0 \\
0 & \mathrm{x} & \mathrm{x} & 0 \\
0 & 0 & \mathrm{x} & \mathrm{x} \\
0 & 0 & 0 & \mathrm{x}
\end{array}\right) \mathbf{G}_{1}\left(\begin{array}{cccc}
\mathrm{x} & 0 & 0 & 0 \\
\mathrm{y} & \mathrm{x} & \mathrm{x} & 0 \\
0 & 0 & \mathrm{x} & \mathrm{x} \\
0 & 0 & 0 & \mathrm{x}
\end{array}\right) \mathbf{G}_{2}\left(\begin{array}{cccc}
\mathrm{x} & \overline{\mathrm{x}} & 0 & 0 \\
0 & \mathrm{x} & \mathrm{x} & 0 \\
0 & 0 & \mathrm{x} & \mathrm{x} \\
0 & 0 & 0 & \mathrm{x}
\end{array}\right) \\
& \mathbf{G}_{3}\left(\begin{array}{cccc}
\mathrm{x} & \overline{\mathrm{x}} & 0 & 0 \\
0 & \mathrm{x} & 0 & 0 \\
0 & \mathrm{y} & \mathrm{x} & \mathrm{x} \\
0 & 0 & 0 & \mathrm{x}
\end{array}\right) \rightarrow\left(\begin{array}{cccc}
\mathrm{x} & \overline{\mathrm{x}} & 0 & 0 \\
0 & \mathrm{x} & \overline{\mathrm{x}} & 0 \\
0 & 0 & \mathrm{x} & \mathrm{x} \\
0 & 0 & 0 & \mathrm{x}
\end{array}\right) \\
& \mathbf{G}_{5}\left(\begin{array}{cccc}
\mathrm{x} & \overline{\mathrm{x}} & 0 & 0 \\
0 & \mathrm{x} & \overline{\mathrm{x}} & 0 \\
0 & 0 & \mathrm{x} & 0 \\
0 & 0 & \mathrm{y} & \mathrm{x}
\end{array}\right) \mathbf{G}_{6}\left(\begin{array}{cccc}
\mathrm{x} & \overline{\mathrm{x}} & 0 & 0 \\
0 & \mathrm{x} & \overline{\mathrm{x}} & 0 \\
0 & 0 & \mathrm{x} & \overline{\mathrm{x}} \\
0 & 0 & 0 & \mathrm{x}
\end{array}\right)
\end{aligned}
$$

where $\mathrm{x}, \mathrm{y}$ and $\overline{\mathrm{x}}$ are nonzero elements (different, in general) of $\mathbf{M}$. Three alternative situations can occur.

(a) If the off-diagonal elements of $\mathbf{M}_{B}^{\prime}$ are zero, or smaller than a predetermined value (tolerance), then $\mathbf{M}$ is a type-I Mueller matrix and

$$
\begin{gathered}
\mathbf{M}_{B}^{\prime}=\mathbf{M}_{(\Delta d)}=\mathbf{G}_{6} \mathbf{G}_{4} \mathbf{G}_{2} \mathbf{U}_{3} \mathbf{U}_{2} \mathbf{U}_{1} \mathbf{M} \mathbf{V}_{1} \mathbf{V}_{2} \mathbf{G}_{1} \mathbf{G}_{3} \mathbf{G}_{5}, \\
\mathbf{M}_{J A}=\left(\mathbf{G}_{6} \mathbf{G}_{4} \mathbf{G}_{2} \mathbf{U}_{3} \mathbf{U}_{2} \mathbf{U}_{1}\right)^{-1}, \\
\mathbf{M}_{J B}=\left(\mathbf{V}_{1} \mathbf{V}_{2} \mathbf{G}_{1} \mathbf{G}_{3} \mathbf{G}_{5}\right)^{-1} .
\end{gathered}
$$

(b) If $b_{12}^{\prime} \approx b_{23}^{\prime} \approx 0$ and $\left|b_{01}^{\prime}\right| \approx|| b_{11}^{\prime}\left|-b_{00}^{\prime}\right|$ (which means that $\mathbf{M}$ is a type-II Mueller matrix), then Eq. (28) also applies for the three components of the normal form, but now the obtained central depolarizer is of type-II, $\mathbf{M}_{B}^{\prime}=\mathbf{M}_{\Delta I I}$.

(c) If none of conditions from the previous cases are met, then the process is iterated until it reaches either case (a) or case (b).

Once the iterations end and the components $\mathbf{M}_{\triangle}$ (namely $\mathbf{M}_{(\Delta d)}$ or $\left.\mathbf{M}_{\Delta I I}\right), \mathbf{M}_{J A}$ and $\mathbf{M}_{J B}$ are calculated, the Mueller matrix components of the normal form for type-I Mueller matrices are obtained from

$$
\begin{aligned}
\mathbf{M}_{\Delta d} & =\mathbf{D}_{2} \mathbf{M}_{(\Delta d)} \mathbf{D}_{1}, \\
\mathbf{M}_{J 2} & =\mathbf{M}_{J B} \mathbf{D}_{2}^{-1}, \\
\mathbf{M}_{J 1} & =\mathbf{D}_{1}^{-1} \mathbf{M}_{J A},
\end{aligned}
$$

where $\mathbf{D}_{1}$ and $\mathbf{D}_{2}$ are appropriate diagonal Mueller matrices $(1,1,-1,-1),(1,-1,1,-1)$ and $(1,-1,-1,1)$ such that the signs of the diagonal elements are set as in the convention taken for the definition of $\mathbf{M}_{\Delta d}$ in Eq. (7).

In the case of type-II Mueller matrices, the matrices of the components are obtained in accordance with Eq. (10).

It should be noted that the two pure matrix components $\mathbf{M}_{J 1}$ and $\mathbf{M}_{J 2}$ can be further decomposed down to products of elliptical diattenuators and retarders by applying the polar decomposition [18]. One thus obtains the symmetric decomposition of $\mathbf{M}$ [4]. Conversely, if the nondepolarizing matrix factors from the symmetric decomposition of $\mathbf{M}$ are multiplied to yield $\mathbf{M}_{J 1}$ and $\mathbf{M}_{J 2}$, one gets the normal form of $\mathbf{M}$.

\section{APPLICATION EXAMPLES}

To illustrate the above algorithm, we apply it to some experimental and numerical type-I and type-II Mueller matrices.

The eigenvalues of $\mathbf{C}(\mathbf{M})$ as well as the values for $D$ (diattenuation), $P$ (polarizance), and $\operatorname{det} \hat{\mathbf{M}}$ are likewise reported since they appear as invariant quantities [19] providing information on the nature of the measured Mueller matrix.

\section{A. Example 1}

The first example considered is the Mueller matrix representing the polarimetric response of a dielectric anechoic coating ("dielectric C") characterized in backscattering geometry [4,20] (the matrix has been pre-multiplied by the reference-frame-change matrix diag $(1,1,-1,-1)$ in order to use a unique reference frame for both input and output beams [4])

$$
\hat{\mathbf{M}}_{1}=\left(\begin{array}{rrrr}
1.0000 & -0.0045 & 0.0172 & 0.0085 \\
-0.0075 & 0.1146 & 0.0018 & 0.0035 \\
-0.0011 & -0.0031 & 0.1079 & -0.0036 \\
-0.0037 & -0.0008 & 0.0069 & -0.0309
\end{array}\right) \text {, }
$$

This Mueller matrix corresponds to a sample exhibiting very small values of diattenuation and polarizance. Its calculated normal form matrix components are

$$
\begin{aligned}
\ldots \ldots \ldots \ldots . . \hat{\mathbf{M}}_{J 1} & =\left(\begin{array}{rrrr}
1.0000 & -0.0037 & 0.0176 & 0.0084 \\
-0.0031 & 0.9990 & 0.0175 & 0.0349 \\
0.0171 & -0.0156 & 0.9983 & -0.0552 \\
0.0095 & -0.0358 & 0.0548 & 0.9977
\end{array}\right), \\
\ldots \ldots \ldots \ldots . . \mathbf{M}_{\Delta d} & =\left(\begin{array}{rrrr}
1.0000 & 0.0000 & 0.0000 & 0.0000 \\
0.0000 & 0.1147 & -0.0007 & 0.0000 \\
0.0000 & -0.0008 & 0.1084 & 0.0000 \\
0.0000 & 0.0000 & 0.0000 & -0.0305
\end{array}\right), \\
& \\
\ldots \ldots \ldots \ldots . . \mathbf{\mathbf { M }}_{J 2} & =\left(\begin{array}{rrrr}
1.0000 & -0.0071 & -0.0033 & -0.0034 \\
-0.0071 & 0.9999 & 0.0064 & 0.0145 \\
-0.0030 & -0.0052 & 0.9967 & -0.0800 \\
-0.0036 & -0.0149 & 0.0799 & 0.9967
\end{array}\right) .
\end{aligned}
$$

Notice that the diagonal type-I depolarizer $\mathbf{M}_{\Delta d}$ is partially degenerate, i.e. its two linear depolarization coefficients are equal. 
In this case both the algorithm described in Ref. [4] for type-I matrices as well as the general algorithm presented in this work produce normal form matrix factors $\hat{\mathbf{M}}_{J 1}$ and $\hat{\mathbf{M}}_{I 2}$ that may contain spurious (apparent) circular birefringence, as discussed in Ref. [0]. The removal of the apparent circular birefringence by applying the procedure described in Ref. [0] reduces the two pure components $\hat{\mathbf{M}}_{J 1}$ and $\hat{\mathbf{M}}_{J 2}$ to essentially horizontal linear retarders.

The corresponding values of $D, P, \operatorname{det} \hat{\mathbf{M}}_{1}$, as well as the eigenvalues $\left(\lambda_{1}, \lambda_{2}, \lambda_{3}, \lambda_{4}\right)$ of $\mathbf{C}\left(\hat{\mathbf{M}}_{1}\right)$ are

$$
\begin{gathered}
D=0.0197, P=0.0084, \operatorname{det} \hat{\mathbf{M}}_{1}=-0.0004, \\
\hat{\lambda}_{1}=0.2987, \hat{\lambda}_{2}=0.2613, \hat{\lambda}_{3}=0.2537, \hat{\lambda}_{4}=0.1863
\end{gathered}
$$

showing that, due to the small values of $D$ and $P$, the device as a whole approximately behaves as a parallel mixture of retarders. Consequently, both pure components $\mathbf{M}_{J 1}$ and $\mathbf{M}_{J 2}$ exhibit very low diattenuation and polarizance values, i.e. are very close to retarder Mueller matrices with weak linear retardances. [Recall that the Mueller matrix of a parallel composition (an incoherent mixture) of retarders features $D=P=0$, exhibits a type-I (diagonal) depolarizer $\mathbf{M}_{\Delta d}$ and its both pure components $\mathbf{M}_{J 1}$ and $\mathbf{M}_{J 2}$ represent elliptical retarders.]

\section{B. Example 2}

As a second example we consider the following Mueller matrix corresponding to a polyacrylamide gel measured in transmission with visible light [22]

$$
\ldots \ldots \ldots . . . . \hat{\mathbf{M}}_{2}=\left(\begin{array}{rrrr}
1.0000 & -0.0312 & 0.0029 & -0.0066 \\
-0.0214 & 0.7678 & -0.0370 & 0.0204 \\
-0.0055 & 0.0230 & 0.1043 & -0.7735 \\
0.0014 & 0.0390 & 0.7972 & 0.1920
\end{array}\right)
$$

whose calculated matrix components are

$$
\begin{aligned}
\ldots \ldots \ldots . . . \hat{\mathbf{M}}_{J 1} & =\left(\begin{array}{rrrr}
1.0000 & -0.0364 & -0.0023 & -0.0304 \\
-0.0245 & 0.0618 & 0.7334 & 0.6757 \\
-0.0357 & 0.9971 & -0.0673 & -0.0169 \\
0.0197 & 0.0324 & 0.6748 & -0.7360
\end{array}\right), \\
\ldots \ldots \ldots \ldots . . . \mathbf{M}_{\Delta d} & =\left(\begin{array}{rrrr}
1.0009 & 0.0000 & 0.0000 & 0.0000 \\
0.0000 & 0.8464 & 0.0000 & 0.0000 \\
0.0000 & 0.0000 & 0.7698 & 0.0001 \\
0.0000 & 0.0000 & 0.0000 & 0.7561
\end{array}\right), \\
\ldots \ldots \ldots . . . \hat{\mathbf{M}}_{J 2} & =\left(\begin{array}{rrrr}
1.0000 & 0.0239 & 0.0058 & -0.0184 \\
0.0071 & 0.0397 & 0.9986 & -0.0196 \\
-0.0280 & -0.5268 & 0.0375 & 0.8491 \\
0.0105 & 0.8488 & -0.0233 & 0.5273
\end{array}\right) .
\end{aligned}
$$

The corresponding values of the invariants considered are

$$
\begin{gathered}
D=0.0320, P=0.0221, \operatorname{det} \hat{\mathbf{M}}_{2}=0.4900, \\
\hat{\lambda}_{1}=0.8424, \hat{\lambda}_{2}=0.0806, \hat{\lambda}_{3}=0.0424, \hat{\lambda}_{4}=0.0346,
\end{gathered}
$$

showing that both pure components $\hat{\mathbf{M}}_{J 1}$ and $\hat{\mathbf{M}}_{J 2}$ correspond closely to retarders, and therefore, $\mathbf{M}_{2}$ is a type-I Mueller matrix (approximately) representable as a parallel mixture of retarders ( $D \approx P \approx 0$ ). It is remarkable that the reported decomposition results coincide exactly with those obtained in [1] by applying the procedure described in Ref. [4].

\section{Example 3}

Consider the following Mueller matrix

$$
\hat{\mathbf{M}}_{3}=\left(\begin{array}{rrrr}
1.0000 & -0.7280 & 0.4274 & 0.3641 \\
-0.1826 & 0.0045 & -0.1432 & -0.2703 \\
-0.2182 & 0.1606 & -0.3152 & 0.0019 \\
0.8471 & -0.7312 & 0.3117 & 0.3482
\end{array}\right),
$$

Its calculated normal matrix components are

$$
\begin{aligned}
& \ldots \ldots \ldots . . . . \hat{\mathbf{M}}_{J 1}=\left(\begin{array}{rrrr}
1.0000 & 0.2308 & -0.2172 & -0.0641 \\
0.3233 & 0.7139 & -0.6716 & -0.1983 \\
0.0000 & -0.2696 & -0.0188 & -0.9069 \\
0.0000 & 0.6053 & 0.7009 & -0.1945
\end{array}\right) \text {, } \\
& \ldots \ldots \ldots . . . . \mathbf{M}_{\Delta n d}=\left(\begin{array}{rrrr}
2.6853 & -1.3427 & -0.0021 & 0.0000 \\
1.3427 & 0.0000 & -0.0034 & 0.0000 \\
0.0000 & 0.0000 & 1.0797 & -0.0002 \\
0.0000 & 0.0000 & 0.0000 & 1.0798
\end{array}\right) \\
& \ldots \ldots \ldots . . . \hat{\mathbf{M}}_{J 2}=\left(\begin{array}{rrrr}
1.0000 & -0.9319 & -0.1479 & -0.2192 \\
-0.1764 & 0.1597 & 0.2552 & -0.0466 \\
-0.3298 & 0.3904 & -0.0398 & -0.1283 \\
0,8935 & -0.8673 & -0.1298 & -0.3019
\end{array}\right) \text {, }
\end{aligned}
$$

with the invariance parameters

$$
\begin{gathered}
D=0.9193, P=0.8936, \operatorname{det} \hat{\mathbf{M}}_{3}=0.0064, \\
\hat{\lambda}_{1}=0.9500, \hat{\lambda}_{2}=0.0320, \hat{\lambda}_{3}=0.0180, \hat{\lambda}_{4}=0.0000 .
\end{gathered}
$$

The inspection of the values shows that $\hat{\mathbf{M}}_{3}$ is close a singular matrix, while it is of type-II with rank $\mathbf{C}\left(\mathbf{M}_{3}\right)=3$ (recall that the rank of the covariance matrix of a type-II Mueller matrix cannot exceed three [5]). It is remarkable that in this case, as well as in all type-II matrices we have processed, the algorithm converges without entering the iteration procedure (i.e., a single iteration is sufficient).

\section{Example 4}

Consider the Mueller matrix

$$
\hat{\mathbf{M}}_{4}=\left(\begin{array}{rrrr}
1.0000 & -0.9444 & 0.0678 & -0.0054 \\
0.8297 & -0.7741 & 0.0678 & -0.0054 \\
0.1049 & -0.1049 & 0.0817 & -0.0528 \\
0.0564 & -0.0564 & 0.0528 & 0.0817
\end{array}\right),
$$

whose calculated normal components are

$$
\ldots \ldots \ldots . . . \hat{\mathbf{M}}_{J 1}=\left(\begin{array}{rrrr}
1.0000 & -0.8766 & 0.0000 & 0.0000 \\
-0.8766 & 1.0000 & 0.0000 & 0.0000 \\
0.0000 & 0.0000 & 0.4813 & 0.0000 \\
0.0000 & 0.0000 & 0.0000 & 0.4813
\end{array}\right) \text {, }
$$

$$
\ldots \ldots \ldots . . . . \mathbf{M}_{\Delta n d}=\left(\begin{array}{rrrr}
0.5856 & -0.2928 & 0.0000 & 0.0001 \\
0.2928 & 0.0000 & 0.0000 & 0.0000 \\
0.0000 & 0.0000 & 0.2927 & 0.0000 \\
0.0000 & 0.0000 & 0.0000 & 0.2927
\end{array}\right) \text {, }
$$


$\ldots \ldots \ldots . . . \hat{\mathbf{M}}_{J 2}=\left(\begin{array}{rrrr}1.0000 & 0.5385 & 0.4814 & -0.0388 \\ 0.6901 & 0.8484 & 0.4814 & -0.0388 \\ 0.1909 & -0.1909 & 0.5799 & -0.3748 \\ 0.1026 & -0.1026 & 0.3748 & 0.5799\end{array}\right)$,

with invariants

$$
\begin{gathered}
D=0.9468, P=0.8382, \operatorname{det} \hat{\mathbf{M}}_{4}=0.0000, \\
\hat{\lambda}_{1}=0.8931, \hat{\lambda}_{2}=0.1069, \hat{\lambda}_{3}=0.0000, \hat{\lambda}_{4}=0.0000 .
\end{gathered}
$$

The inspection of the decomposition results shows that $\hat{\mathbf{M}}_{4}$ is close to a singular matrix, while it is clearly of type-II with $\operatorname{rank} \mathbf{C}\left(\mathbf{M}_{4}\right)=2$. Notice that the pure component $\mathbf{M}_{J 2}$ represents a horizontal diattenuator. Because of $\operatorname{rank} \mathbf{C}\left(\mathbf{M}_{4}\right)=2$, $\mathbf{M}_{4}$ can be represented as the parallel combination of two pure components: a polarize्रr-analyzer and a waveplate [23]. Finally, observe that matrices $\hat{\mathbf{M}}_{i}(i=1,2,3,4)$ of the above examples as well as the serial matrix components of their normal forms can be further normalized to comply with the passivity criterion as described in Ref. [24].

\section{CONCLUSIONS}

An original algorithm allowing for the numerical calculation of the matrix components of the normal form of an experimentally determined Mueller matrix has been presented. The procedure efficiently provides the normal components of type-II Mueller matrices, besides those of type I, thus covering the complete range of possible types of measured Mueller matrices, including singular ones.

The algorithm has been illustrated on a number of experimental and numerical Mueller matrices, of both type I and type II. It is believed to be of use to experimentalists willing to interpret physically their measurements in phenomenological terms.

It should be noted before concluding that, prior to the application of the algorithm, it is advisable to check the covariance condition (i.e., the nonnegativity of the eigenvalues of the coherency matrix associated with the Mueller matrix under analysis) and, whenever appropriate, to apply a filtering procedure [25-29].

\section{Appendix A}

The explicit forms of the Givens matrices used in the algorithm are reported below.

$$
\mathbf{G}_{1}=\left(\begin{array}{cccc}
\cosh \theta & -\sinh \theta & 0 & 0 \\
-\sinh \theta & \cosh \theta & 0 & 0 \\
0 & 0 & 1 & 0 \\
0 & 0 & 0 & 1
\end{array}\right)
$$

$$
\begin{aligned}
\cosh \theta= & \frac{b_{00}}{\sqrt{b_{00}^{2}-b_{01}^{2}}}, \sinh \theta=\frac{b_{01}}{\sqrt{b_{00}^{2}-b_{01}^{2}}} . \\
\mathbf{G}_{2} & =\left(\begin{array}{cccc}
\cosh \theta & -\sinh \theta & 0 & 0 \\
-\sinh \theta & \cosh \theta & 0 & 0 \\
0 & 0 & 1 & 0 \\
0 & 0 & 0 & 1
\end{array}\right),
\end{aligned}
$$

$$
\cosh \theta=\frac{b_{00}}{\sqrt{b_{00}^{2}-b_{10}^{2}}}, \sinh \theta=\frac{b_{10}}{\sqrt{b_{00}^{2}-b_{10}^{2}}} .
$$

$$
\mathbf{G}_{3}=\left(\begin{array}{cccc}
1 & 0 & 0 & 0 \\
0 & \cos \theta & -\sin \theta & 0 \\
0 & \sin \theta & \cos \theta & 0 \\
0 & 0 & 0 & 1
\end{array}\right),
$$

$$
\cos \theta=\frac{b_{11}}{\sqrt{b_{11}^{2}+b_{12}^{2}}}, \sin \theta=\frac{b_{12}}{\sqrt{b_{11}^{2}+b_{12}^{2}}} .
$$

$$
\mathbf{G}_{4}=\left(\begin{array}{cccc}
1 & 0 & 0 & 0 \\
0 & \cos \theta & \sin \theta & 0 \\
0 & -\sin \theta & \cos \theta & 0 \\
0 & 0 & 0 & 1
\end{array}\right)
$$

$$
\cos \theta=\frac{b_{11}}{\sqrt{b_{11}^{2}+b_{21}^{2}}}, \sin \theta=\frac{b_{21}}{\sqrt{b_{11}^{2}+b_{21}^{2}}} .
$$

$$
\mathbf{G}_{5}=\left(\begin{array}{cccc}
1 & 0 & 0 & 0 \\
0 & 1 & 0 & 0 \\
0 & 0 & \cos \theta & -\sin \theta \\
0 & 0 & \sin \theta & \cos \theta
\end{array}\right)
$$

$$
\begin{gathered}
\cos \theta=\frac{b_{22}}{\sqrt{b_{22}^{2}+b_{23}^{2}}}, \sin \theta=\frac{b_{23}}{\sqrt{b_{22}^{2}+b_{23}^{2}}} . \\
\mathbf{G}_{6}=\left(\begin{array}{cccc}
1 & 0 & 0 & 0 \\
0 & 1 & 0 & 0 \\
0 & 0 & \cos \theta & \sin \theta \\
0 & 0 & -\sin \theta & \cos \theta
\end{array}\right), \\
\cos \theta=\frac{b_{22}}{\sqrt{b_{22}^{2}+b_{32}^{2}}}, \sin \theta=\frac{b_{32}}{\sqrt{b_{22}^{2}+b_{32}^{2}}} .
\end{gathered}
$$

Disclosures. The authors declare no conflicts of interest.

\section{References}

1. J. J. Gil and R. Ossikovski, Polarized Light and the Mueller Matrix Approach. (CRC Press 2016).

2. R. Sridhar and R. Simon, "Normal form for Mueller matrices in polarization optics, "J. Mod. Opt. 41, 1903-1915 (1994).

3. R. Ossikovski, M. Anastasiadou, S. Ben Hatit, E. Garcia-Caurel, and A. De Martino, "Depolarizing Mueller matrices: how to decompose them?," phys. stat. sol. (a) 205, 720-727 (2008).

4. R. Ossikovski, "Analysis of depolarizing Mueller matrices through a symmetric decomposition," J. Opt. Soc. Am. A 26, 11091118 (2009).

5. R. Ossikovski, "Canonical forms of depolarizing Mueller matrices," J. Opt. Soc. Am. A 27, 123-130 (2010).

6. Z.-F. Xing, "On the deterministic and non-deterministic Mueller matrix," J. Mod. Opt. 39, 461-484 (1992).

7. S.-Y. Lu and R. A. Chipman, "Interpretation of Mueller matrices based on polar decomposition," J. Opt. Soc. Am. A 13, 1106-1113 (1996).

8. S. R. Cloude, "Group theory and polarization algebra", Optik 75, 26-36 (1986).

9. J. J. Gil, "Characteristic properties of Mueller matrices," J. Opt. Soc. Am. A 17, 328-334 (2000).

10. J. J. Gil, "Polarimetric characterization of light and media," Eur. Phys. J. Appl. Phys. 40, 1-47 (2007).

11. J. J. Gil and I. San José, "Polarimetric subtraction of Mueller matrices," J. Opt. Soc. Am. A 30, 1078-1088 (2013). 
12.S. R. Cloude, Polarisation: Applications in Remote Sensing, (Oxford University Press, 2009).

13. Y. Bolshakov, C. V. M. van der Mee, and A. C. M. Ran, "Polar decompositions in finite dimensional indefinite scalar product spaces: special cases and applications," In Operator Theory: Advances and Applications, I. Gohberg, ed. Birkhäuser Verlag, 87, 61-94 (1996).

14. Y. Bolshakov, C. V. M. van der Mee, and A. C. M. Ran, "Errata for: Polar decompositions in finite dimensional indefinite scalar product spaces: special cases and applications," Integral Equation Oper. Theory 27, 497501 (1997).

15. A. V. Gopala Rao, S. Mallesh, and Sudha Shenoy, "On the algebraic characterization of a Mueller matrix in polarization optics. I. Identifying a Mueller matrix from its N-matrix," J. Mod. Opt. 45, 955-987 (1998).

16. A. V. Gopala Rao, K. S. Mallesh, and Sudha Shenoy, "On the algebraic characterization of a Mueller matrix in polarization optics. II. Necessary and sufficient conditions for Jones derived Mueller matrices," J. Mod. Opt. 45, 989-999 (1998)

17. J. J. Gil, R. Ossikovski, and I. San José, "Singular Mueller matrices," J. Opt. Soc. Am. A 33, 600-609 (2016).

18. J. J. Gil and E. Bernabéu, "Obtainment of the polarizing and retardation parameters of a nondepolarizing optical system from its Mueller matrix," Optik 76, 67-71 (1987).

19. J. J. Gil, "Invariant quantities of a Mueller matrix under rotation and retarder transformations," J. Opt. Soc. Am. A 33, 52-58 (2016).

20. F. Le Roy-Bréhonnet and B. Le Jeune, "Utilization of Mueller matrix formalism to obtain optical targets depolarization and polarization properties," Prog. Quantum Electron. 21, 109-151 (1997).
21. J. Vizet and R. Ossikovski, "Symmetric decomposition of experimental depolarizing Mueller matrices in the degenerate case," Appl. Opt. 57, 1159-1167 (2018).

22. O. Arteaga and A. Canillas, "Pseudopolar decomposition of the Jones and Mueller-Jones exponential polarization matrices," J. Opt. Soc. Am. A. 26 783-793 (2009).

23. R. Ossikovski and J. J. Gil, "Basic properties and classification of Mueller matrices derived from their statistical definition," J. Opt. Soc. Am. A 34 1727-1737 (2017).

24. I. San José and J. J. Gil, "Characterization of passivity in Mueller matrices," J. Opt. Soc. Am. A 37, 199-208 (2020).

25. A. Aiello, G. Puentes, D. Voigt, and J. P. Woerdman, "Maximum-likelihood estimation of Mueller matrices," Opt. Lett. 31, 817-819 (2006).

26. R. Ossikovski, "Retrieval of a nondepolarizing estimate from an experimental Mueller matrix through virtual experiment, "Opt. Lett. 37, 578-580 (2012).

27. S. Faisan, C. Heinrich, G. Sfikas, and J. Zallat, "Estimation of Mueller matrices using non-local means filtering," Opt. Express 21, 4424-4438 (2013).

28. J. J. Gil, "On optimal filtering of measured Mueller matrices," Appl. Opt. 55 5449-5455 (2016).

29. R. Ossikovski and O. Arteaga, "Instrument-dependent method for obtaining a nondepolarizing estimate from an experimental Mueller matrix," Opt. Eng. 58, 082409 (2019). 